04,05, 10,09

\title{
Расчет электронной структуры, динамики решетки, оптических и магнитных свойств тетрабората европия $\mathrm{EuB}_{4} \mathrm{O}_{7}$
}

\author{
() А.С. Шинкоренко, М.С. Павловский, В.И. Зиненко
}

Институт ффизики им. Л.В. Киренского СО РАН,

Красноярск, Россия

E-mail: shas@iph.krasn.ru

(Поступила в Редакцию 23 марта 2016 г.)

В рамках метода функционала плотности проведены расчеты электронной зонной структуры, частот колебаний кристаллической решетки, оптических и магнитных свойств кристалла $\mathrm{EuB}_{4} \mathrm{O}_{7}$ со структурой типа $\alpha-\mathrm{SrB}_{4} \mathrm{O}_{7}$. Обнаружено, что данное соединение является диэлектриком с шириной запрещенной зоны порядка $4 \mathrm{eV}$. Установлено, что основным состоянием для указанного кристалла является состояние с ферромагнитным упорядочением спинов $\mathrm{Eu}^{2+}$. Вычислены константы обменного взаимодействия, в рамках приближения молекулярного поля проведена оценка температуры ферромагнитного упорядочения $\left(T_{c} \approx 1 \mathrm{~K}\right)$. Соединение $\mathrm{EuB}_{4} \mathrm{O}_{7}$ является магнитным пироэлектриком и, следовательно, может проявлять магнитоэлектрические свойства. Вычисленная величина изменения поляризации при ферромагнитном упорядочении для данного кристалла составила $3973 \mu \mathrm{C} / \mathrm{m}^{2}$.

Работа выполнена при финансовой поддержке РФФИ (грант № 16-32-00373мол_а) и программы СО РАН № II.2П.

Результаты работы получены с использованием вычислительных ресурсов МВК НИЦ „Курчатовский институт“(http://computing.kiae.ru/).

\section{1. Введение}

Соединения тетраборатов с общей химической формулой $A \mathrm{~B}_{4} \mathrm{O}_{7}$ (где $A-$ двухвалентный элемент) являются перспективными материалами для применения в лазерной технике. Отличительными особенностями данных соединений являются широкое окно прозрачности в УФ- и оптической частях спектра, относительно высокие значения нелинейно-оптических коэффициентов, стойкость к оптическому, химическому и физическому воздействиям. В зависимости от состава и условий роста в соединениях тетраборатов могут реализоваться различные структурные модификации, причем физические свойства этих модификаций могут значительно различаться. Особое внимание исследователей уделяется соединениям, изоморфным $\alpha$-модификации тетрабората стронция с полярной ромбической пространственной группой, в которых наблюдаются большие величины второй оптической гармоники, что наряду с широким окном оптической прозрачности делает такие материалы перспективными для приложений в нелинейной оптике $[1,2]$. Среди кристаллов, изоморфных $\alpha$-модификации тетрабората стронция, $\mathrm{EuB}_{4} \mathrm{O}_{7}$ (ЕВО), по-видимому, является единственным известным соединением, содержащим магнитный ион, что делает его привлекательным с точки зрения изучения нелинейных оптических, магнитооптических и магнитоэлектрических свойств. Однако в литературе имеется крайне мало работ, посвященных исследованию этого соединения. В [3,4] приводятся данные о структуре $\mathrm{EuB}_{4} \mathrm{O}_{7}$ и частотах колебаний решетки, активных в ИК-области, а также результаты измерения магнитной восприимчивости.
В настоящей работе приводятся результаты расчетов из первых принципов динамики кристаллической решетки, электронной зонной структуры, магнитных и оптических свойств кристалла ЕВО.

\section{2. Метод расчета}

Вычисления выполнялись с помощью пакета VASP („The Vienna Ab initio Simulation Package“) [5,6] c использованием метода присоединенных плоских волн (PAW) [7,8]. Функционал обменно-корреляционной энергии электронов учитывался с использованием обобщенного градиентного приближения (GGA-PBE) [9]. Конфигурация валентных электронов для ионов $\mathrm{Eu}-4 f^{7} 5 s^{2} 5 p^{6} 6 s^{2}$, для ионов $\mathrm{B}-2 s^{2} 2 p^{1}$, для ионов $\mathrm{O}-2 s^{2} 2 p^{4}$. Число плоских волн ограничивалось энергией $600 \mathrm{eV}$, сеть Монкхорста-Пака [10] при оптимизации параметров элементарной ячейки была выбрана равной $4 \times 9 \times 9$, для вычисления электронной зонной структуры $-9 \times 22 \times 23$, при расчете магнитных свойств для увеличенной ячейки $1 \times 2 \times 2$ сеть Монкхорста-Пака выбиралась равной $4 \times 4 \times 5$. Для учета сильных корреляций $f$-электронов ионов $\mathrm{Eu}$ использовался метод $\mathrm{GGA}+U$ в приближении Дударева [11]. Оптимизация параметров и координат атомов проводилась до тех пор, пока остаточные силы, действующие на ионы, не становились меньше $1 \mathrm{meV} / \AA$. Расчет проводился при нескольких значениях параметра $U$ для иона $\mathrm{Eu}(U=2-8 \mathrm{eV})$. Результаты расчета динамики решетки и магнитных свойств ЕВО оказались малочувствительными к величине $U$. Более чувствитель- 


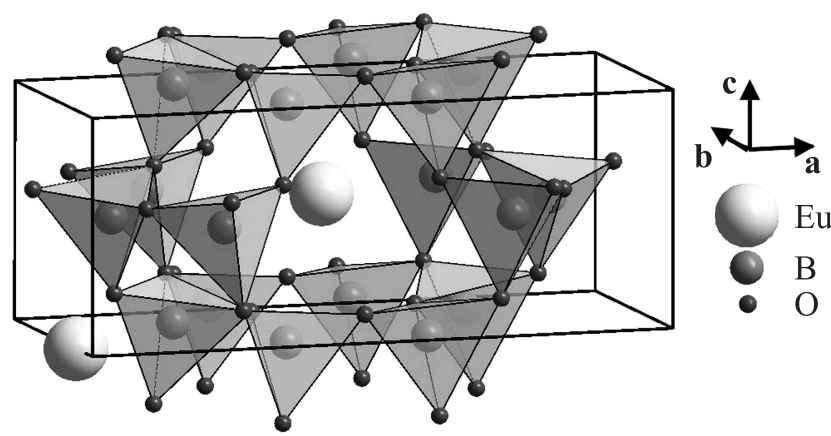

Pис. 1. Структура кристалла тетрабората европия с пространственной группой симметрии $P m n 2_{1}$.

ны к величине $U$ оптические свойства, в частности коэффициент поглощения электромагнитных волн. При $U=4 \mathrm{eV}$ имеется наилучшее согласие вычисленного и экспериментального коэффициентов абсорбции. В связи с этим представленные далее результаты расчетов соответствуют величине $U=4 \mathrm{eV}$.

\section{3. Структура}

Кристалл ЕВО обладает структурой с полярной пространственной группой симметрии $P m n 2_{1}$ [4] и представляет собой трехмерный каркас, состоящий из соединенных вершинами бор-кислородных тетраэдров $\mathrm{BO}_{4}$, в полостях которого располагаются ионы европия (рис. 1).

Элементарная ячейка содержит две молекулярные единицы. Вычисленные без учета спиновой поляризации равновесные параметры решетки и относительные координаты атомов представлены в табл. 1. Там же для сравнения приведены экспериментальные значения, полученные в работе [4]. Как видно из табл. 1, расчетные данные находятся в хорошем согласии с экспериментом.

\section{4. Динамика решетки}

Для полученной равновесной структуры ЕВО был проведен расчет динамики кристаллической решетки. Вычисленные частоты колебаний в центре зоны Бриллюэна представлены в табл. 2. Там же для сравнения приведены экспериментальные значения частот мод колебаний, активных в ИК-спектре, из работы [3].

Разложение колебательного представления по неприводимым представлениям в центре зоны Бриллюэна имеет вид $\Gamma=19 A_{1}+17 A_{2}+17 B_{1}+19 B_{2}$ (включая акустические моды $\left.A_{1}+B_{1}+B_{2}\right)$. Среди оптических мод полярными являются моды $A_{1}, B_{1}, B_{2}$, они активны в рамановских спектрах и ИК-спектрах. Неполярные $A_{2}$-моды являются только раман-активными. Набор экспериментальных значений ИК-активных частот колебаний в табл. 2 является неполным (частоты с величиной ниже $400 \mathrm{~cm}^{-1}$ не наблюдались), и частоты не классифицированы по неприводимым представлениям (в ра- боте [3] исследования осуществлялись на поликристаллических образцах), поэтому сравнивать их напрямую с вычисленными частотами не представляется возможным. Можно только отметить, что ИК-активные частоты колебаний с близкими к экспериментальным значениями среди вычисленных присутствуют. Исключением

Таблица 1. Параметры ячейки и относительные координаты атомов кристалла тетрабората европия (пространственная группа симметрии $\left.P m n 2_{1}\right)$, полученные экспериментально в работе [4] и вычисленные в настоящей работе (в скобках)

\begin{tabular}{|c|c|c|c|c|c|c|}
\hline \multicolumn{3}{|c|}{ Параметры ячейки, А̊ } & \multirow{2}{*}{ Атом } & \multicolumn{3}{|c|}{ Координаты атомов } \\
\hline$a$ & $b$ & $c$ & & $x / a$ & $y / b$ & $z / c$ \\
\hline \multirow{7}{*}{$\begin{array}{c}10.731 \\
(10.805)\end{array}$} & \multirow{7}{*}{$\begin{array}{c}4.435 \\
(4.461)\end{array}$} & \multirow{7}{*}{$\begin{array}{c}4.240 \\
(4.267)\end{array}$} & $\mathrm{Eu}$ & $\begin{array}{c}0.0000 \\
(0.0000)\end{array}$ & $\begin{array}{c}0.2116 \\
(0.2116)\end{array}$ & $\begin{array}{c}0.0000 \\
(0.0000)\end{array}$ \\
\hline & & & B1 & $\begin{array}{c}0.1220 \\
(0.1215)\end{array}$ & $\begin{array}{c}0.6720 \\
(0.6650)\end{array}$ & $\begin{array}{c}0.4630 \\
(0.4955)\end{array}$ \\
\hline & & & B2 & 0.2510 & 0.1780 & 0.484 \\
\hline & & & & $(0.2514)$ & $(0.1677)$ & $(0.4712)$ \\
\hline & & & $\mathrm{O} 1$ & $\begin{array}{c}0.0000 \\
(0.0000)\end{array}$ & $\begin{array}{c}0.7680 \\
(0.7601)\end{array}$ & $\begin{array}{c}0.5810 \\
(0.5805)\end{array}$ \\
\hline & & & $\mathrm{O} 2$ & $\begin{array}{c}0.1410 \\
(0.1418)\end{array}$ & $\begin{array}{c}0.3580 \\
(0.3476)\end{array}$ & $\begin{array}{c}0.5460 \\
(0.5414)\end{array}$ \\
\hline & & & $\mathrm{O} 3$ & $\begin{array}{c}0.1350 \\
(0.1350)\end{array}$ & $\begin{array}{c}0.7270 \\
(0.7199)\end{array}$ & $\begin{array}{c}0.1340 \\
(0.1388)\end{array}$ \\
\hline
\end{tabular}

Таблица 2. Вычисленные в настоящей работе и полученные экспериментально в работе [3] частоты колебаний $\left(\mathrm{cm}^{-1}\right)$ кристаллической решетки кристалла ЕВО

\begin{tabular}{r|r|r|r|c}
\hline \multicolumn{1}{c|}{$A_{1}$} & $A_{2}$ & $B_{1}$ & $B_{2}$ & Данные [3] \\
\hline 1123 & 1133 & 1181 & 1304 & 1450 \\
1082 & 1073 & 1041 & 1046 & 1310 \\
1020 & 1043 & 993 & 1036 & 1222 \\
971 & 976 & 957 & 948 & 1192 \\
874 & 914 & 915 & 937 & 1160 \\
791 & 784 & 869 & 876 & 1100 \\
732 & 740 & 781 & 793 & 1020 \\
706 & 713 & 773 & 780 & 970 \\
639 & 651 & 651 & 717 & 905 \\
621 & 608 & 647 & 611 & 886 \\
574 & 535 & 595 & 543 & 812 \\
485 & 504 & 505 & 511 & 777 \\
431 & 420 & 447 & 444 & 725 \\
368 & 337 & 294 & 316 & 705 \\
311 & 280 & 211 & 305 & 658 \\
289 & 217 & 135 & 275 & 644 \\
127 & 116 & & 114 & 626 \\
68 & & & 83 & 554 \\
& & & & 532 \\
& & & & \\
& & & & \\
& & & & 470 \\
& & & &
\end{tabular}


является колебание с частотой $1450 \mathrm{~cm}^{-1}$, наблюдаемое экспериментально и отсутствующее в данном расчете. Столь высокие частоты колебаний свойственны соединениям боратов, в структурах которых присутствуют боркислородные треугольники $\mathrm{BO}_{3}$ [12]. В изоструктурных кристаллах $\mathrm{SrB}_{4} \mathrm{O}_{7}$ и $\mathrm{PbB}_{4} \mathrm{O}_{7}$ при теоретических расчетах и экспериментальных исследованиях рамановских и ИК-спектров $[13,14]$ колебаний с такими высокими частотами обнаружено не было.

\section{5. Зонная структура}

Для исследуемого соединения был проведен расчет зонной структуры как с учетом, так и без учета спиновой поляризации. На рис. 2 показаны результаты расчета дисперсионных зависимостей энергий электронных состояний с учетом спиновой поляризации для всех симметричных направлений зоны Бриллюэна пространственной группы симметрии Pmn $2_{1}$. Там же справа показана плотность электронных состояний. Из рис. 2 можно видеть, что ширина запрещенной зоны тетрабората европия составляет величину порядка $4 \mathrm{eV}$. Полученное значение ширины запрещенной зоны согласуется с экспериментально наблюдаемым явлением люминесценции в тетраборате европия [4], которое связано с переходом электронов $4 f^{7} \rightarrow 4 f^{6} 5 d^{1}$ и характеризуется испускаемым светом с длиной волны $\lambda=370 \mathrm{~nm}$ (что соответствует энергии $E=3.35 \mathrm{eV})$.

Парциальные плотности электронных состояний представлены на рис. 3. Ввиду малых значений плотность состояний для $s$ - и $p$-электронов иона Еu не приводится. Видно, что узкие пики в плотности состояний вблизи уровня Ферми для конфигурации „спин вверх““ в валентной зоне и на верхней границе зоны проводимости в конфигурации „спин вниз“ соответствуют $f$-электронам европия.

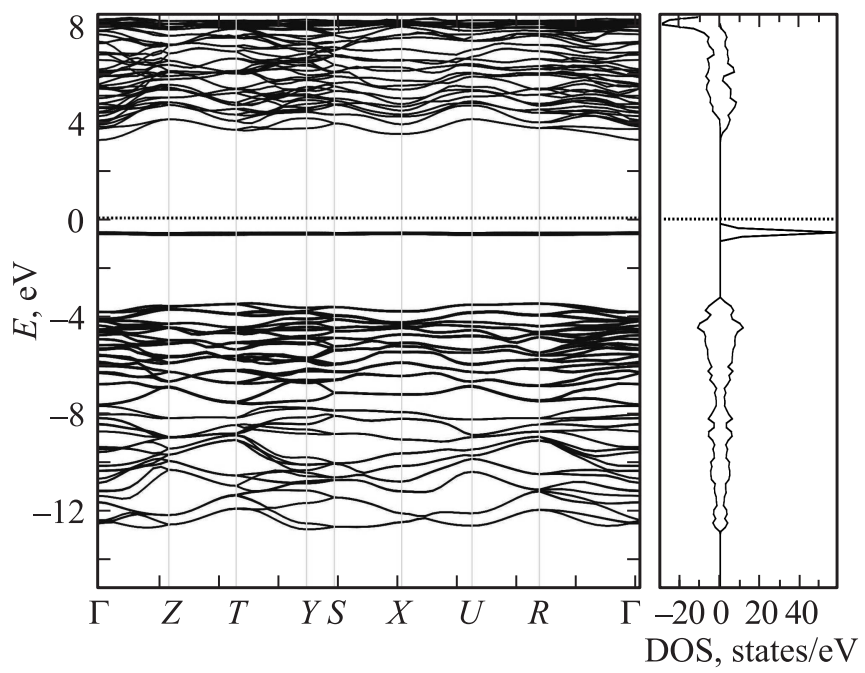

Pис. 2. Электронная зонная структура и плотность состояний (DOS) тетрабората европия при расчете с учетом спиновой поляризации.

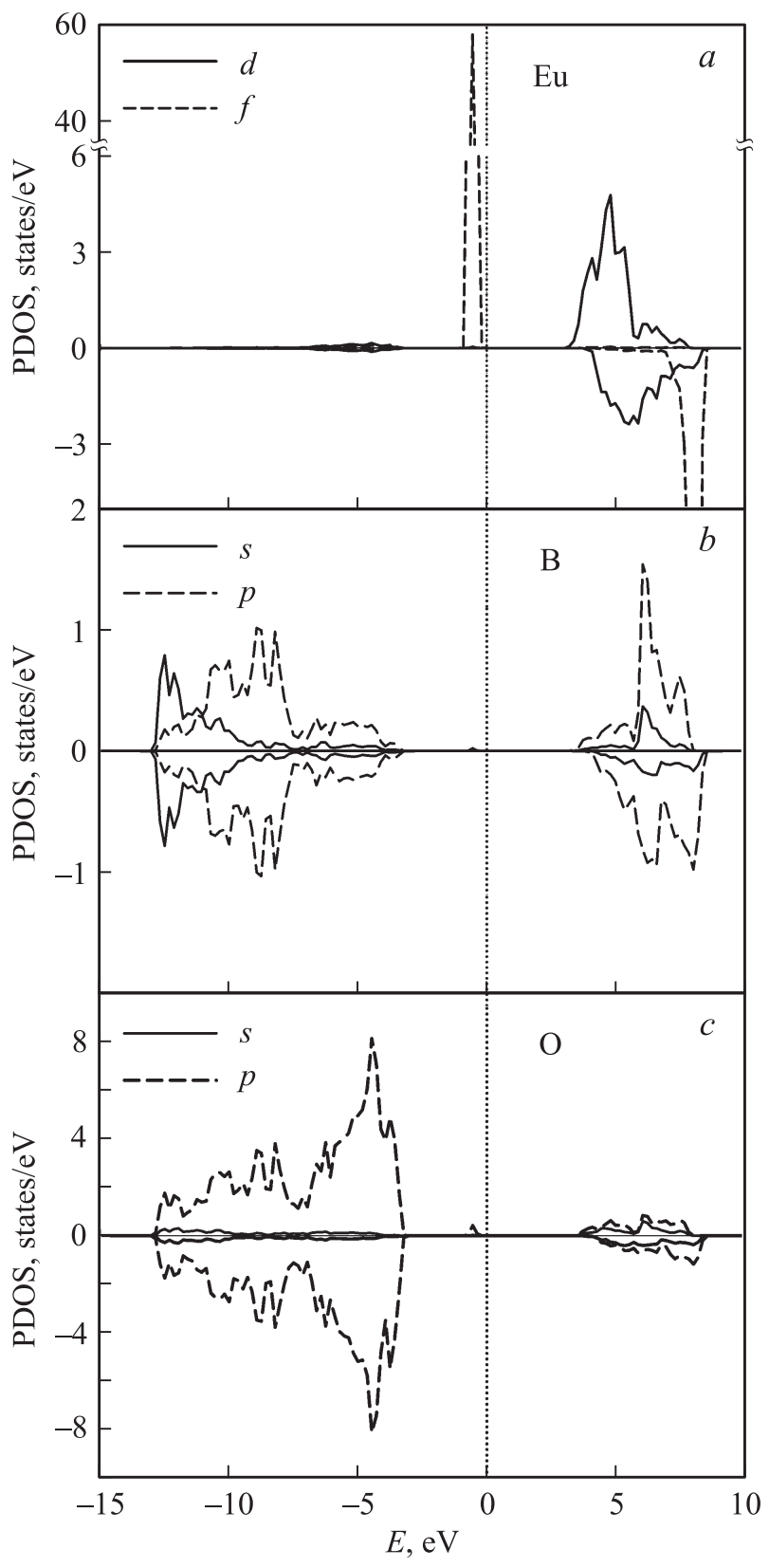

Рис. 3. Парциальные плотности электронных состояний (PDOS) ионов $\mathrm{Eu}(a), \mathrm{B}(b)$ и $\mathrm{O}(c)$.

Как видно из рис. 2 и 3, узкая полоса состояний $f$-электронов европия в валентной зоне отделена от состояний остальных валентных электронов большой энергетической щелью порядка $3.5 \mathrm{eV}$. Подобные особенности зонной структуры ранее наблюдались в других работах при расчете зонной структуры соединений, содержащих $\mathrm{Eu}^{2+}[15,16]$.

При расчете без учета спиновой поляризации пик $f$-электронов на плотности электронных состояний смещается ко дну зоны проводимости, и запрещенная зона становится порядка $1 \mathrm{eV}$, причем энергетическая щель между состояниями $f$-электронов и состояниями остальных электронов в валентной зоне увеличивается до $6 \mathrm{eV}$. 


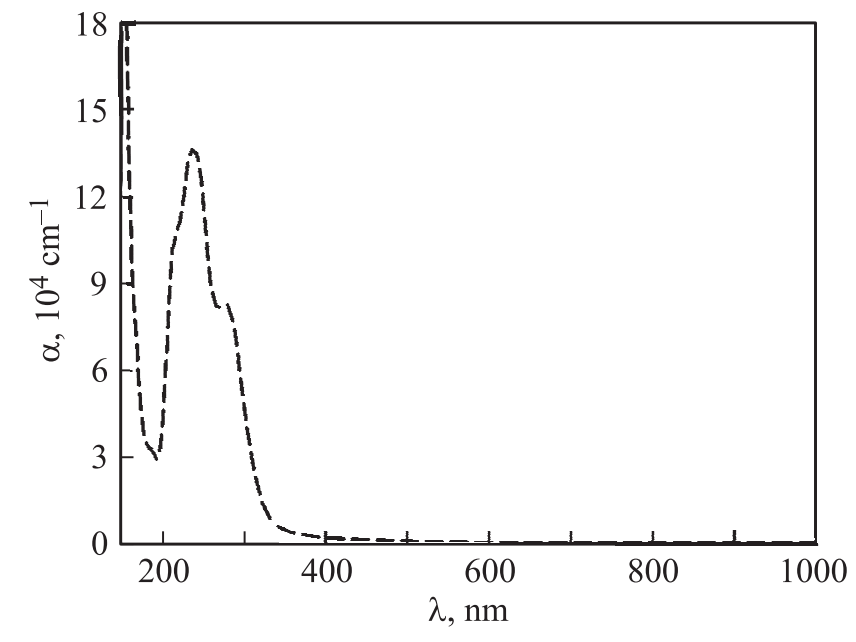

Рис. 4. Вычисленная зависимость среднего коэффициента поглощения от длины волны.

\section{6. Оптические свойства}

Для тетрабората европия была вычислена зависимость диэлектрической функции от частоты [17]. Зависимость коэффициента поглощения $\alpha$ от частоты вычислялись по формуле

$$
\alpha(\omega)=\frac{2 \omega}{c} \sqrt{\frac{|\varepsilon(\omega)|-\varepsilon^{\prime}(\omega)}{2}},
$$

где $\varepsilon(\omega)$ - комплексная диэлектрическая проницаемость, $\varepsilon^{\prime}(\omega)$ - действительная часть диэлектрической проницаемости. Зависимость коэффициента поглощения от длины волны представлена на рис. 4. Как видно из этого рисунка, коэффициент поглощения имеет два максимума при длинах волн 240 и $280 \mathrm{~nm}$. Экспериментальных данных по исследованию оптических свойств EBO в литературе найдено не было. Однако следует отметить, что при экспериментальном исследовании поглошения изоструктурного кристалла $\mathrm{SrB}_{4} \mathrm{O}_{7}$, допированного 5\% $\mathrm{Eu}^{2+}[18]$, наблюдаются пики поглощения света при длинах волн приблизительно 248 и $300 \mathrm{~nm}$. По экспериментальным данным для пропускания света в недопированном кристалле $\mathrm{SrB}_{4} \mathrm{O}_{7}[19]$ в диапазоне длин волн от 130 до $300 \mathrm{~nm}$ таких пиков поглощения обнаружено не было, и можно сделать вывод о том, что в случае допированного $\mathrm{SrB}_{4} \mathrm{O}_{7}$ появление пиков поглощения обусловлено наличием ионов европия. Как уже отмечалось, вычисляемые оптические свойства оказываются чувствительными к величине параметра $U$. Например, для значений $U=2 \mathrm{eV}$ максимумы пиков поглощения смещаются в область длинных волн (290 и $356 \mathrm{~nm})$. При значении $U=6 \mathrm{eV}$ максимумы пиков смещаются в область коротких волн (200 и $232 \mathrm{~nm})$.

\section{7. Магнитные свойства}

Ион двухвалентного европия является магнитным со спином $s=7 / 2$. С целью определения основного магнитного состояния и параметров обменного взаимодействия в тетраборате европия был проведен расчет полных энергий кристалла с учетом спиновой поляризации для различных магнитных упорядоченных структур. Оценка обменных констант взаимодействия была проведена в рамках классической модели Гейзенберга

$$
H=-\frac{1}{2} \sum_{i, j} J_{i j} \hat{S}_{i} \hat{S}_{j},
$$

где $J_{i j}$ - константы обменного взаимодействия между узлами $i$ и $j, \hat{S}_{i}$ и $\hat{S}_{j}$ - операторы спина на узлах $i$ и $j$ соответственно.

Для определения основного магнитного состояния и величин обменных взаимодействий использовалась увеличенная в 4 раза ячейка с удвоением параметров $b$ и $c$, содержащая восемь ионов европия. Система магнитных ионов $\mathrm{Eu}^{2+}$ разбивалась на восемь подрешеток, и учитывались взаимодействия в пределах четырех координационных сфер, как это показано на рис. 5.

На рис. 6 схематически представлены рассматриваемые в настоящей работе магнитные структуры в описанной выше учетверенной ячейке. Для каждой такой магнитной структуры были вычислены значения полной энергии кристалла. В табл. 3 приведены выражения для

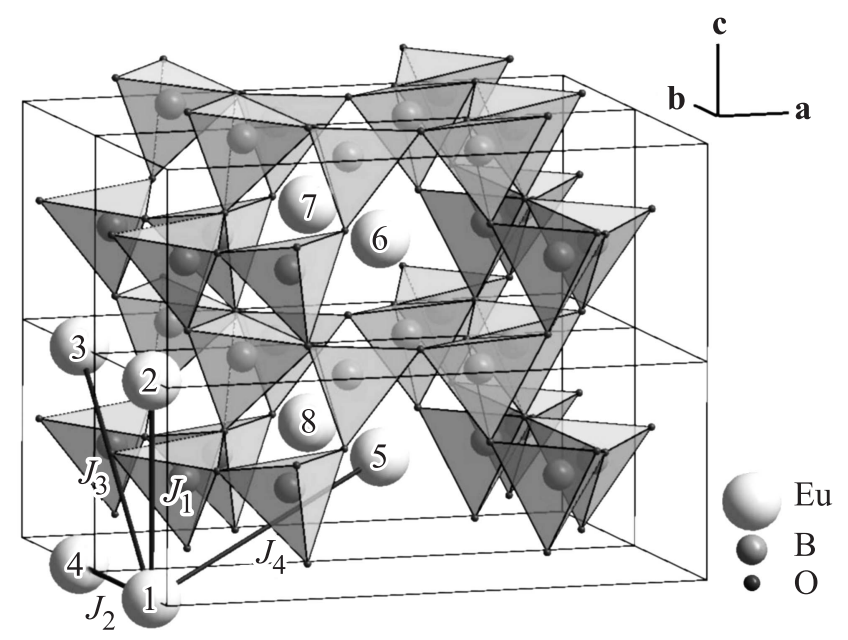

Рис. 5. Увеличенная ячейка тетрабората европия $1 \times 2 \times 2$ с обозначением магнитных ионов и обменных констант.

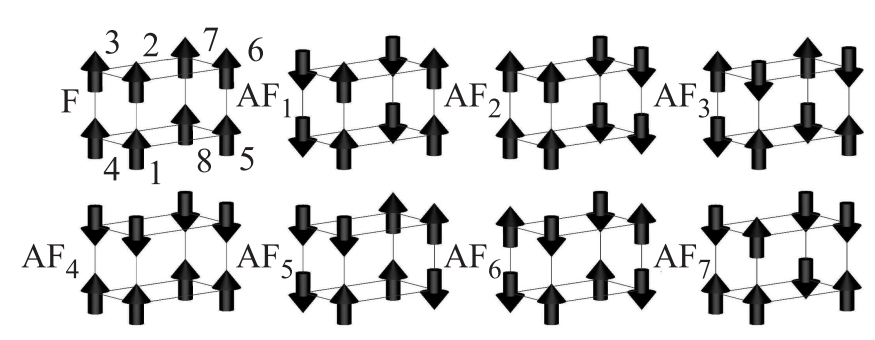

Рис. 6. Схематическое изображение рассматриваемых магнитных конфигураций ЕВО. 
Таблица 3. Выражения для энергий магнитных конфигураций через обменные константы и величины $\Delta E_{i}$

\begin{tabular}{c|c|c}
\hline $\begin{array}{c}\text { Магнитная } \\
\text { структура } \\
\text { (см. рис. 6) }\end{array}$ & $\Delta E_{i}, \mathrm{meV}$ & $\begin{array}{c}\text { Выражения для энергий } \\
\text { на основе } \\
\text { гамильтониана (2) }\end{array}$ \\
\hline $\mathrm{F}$ & 0 & $8 J_{1}+8 J_{2}+16 J_{3}+32 J_{4}$ \\
$\mathrm{AF}$ & 1.80 & $8 J_{1}-8 J_{2}-16 J_{3}$ \\
$\mathrm{AF}_{2}$ & 0.51 & $8 J_{1}+8 J_{2}+16 J_{3}-32 J_{4}$ \\
$\mathrm{AF}_{3}$ & 0.94 & $-8 J_{1}-8 J_{2}+16 J_{3}$ \\
$\mathrm{AF}_{4}$ & 1.34 & $-8 J_{1}+8 J_{2}-16 J_{3}$ \\
$\mathrm{AF}_{5}$ & 1.26 & $-4 J_{4}$ \\
$\mathrm{AF}_{6}$ & 0.97 & $-8 J_{1}-8 J_{2}+16 J_{3}$ \\
$\mathrm{AF}_{7}$ & 1.13 & $-4 J_{4}$ \\
\end{tabular}

энергий магнитных конфигураций через обменные константы гамильтониана (2) и значения $\Delta E_{i}=E_{\mathrm{A} F_{1}}-E_{\mathrm{F}}$, где $E_{\mathrm{F}}$ - полная энергия кристалла при ферромагнитном упорядочении, $E_{\mathrm{A} F_{i}}-$ полная энергия кристалла при конкретном антиферромагнитном упорядочении $i$-го типа. Видно, что наиболее энергетически выгодным в тетраборате европия является ферромагнитное упорядочение.

Вычисленные значения обменных констант составили $J_{1}=-1.4 \cdot 10^{-5} \mathrm{eV}, J_{2}=3.9 \cdot 10^{-5} \mathrm{eV}, J_{3}=5.3 \cdot 10^{-5} \mathrm{eV}$, $J_{4}=0.7 \cdot 10^{-5} \mathrm{eV}$.

Температура фазового перехода в ферромагнитное состояние оценивалась в приближении молекулярного поля, в котором выражения для средних спинов подрешеток $\left\langle S_{i}\right\rangle$ имеют вид

$$
\begin{aligned}
\left\langle S_{1}\right\rangle= & B_{S_{1}}\left(2 J_{1} S_{4}+2 J_{2} S_{2}+4 J_{3} S_{3}\right. \\
& \left.+2 J_{4}\left(S_{5}+S_{6}+S_{7}+S_{8}\right)\right), \\
\left\langle S_{5}\right\rangle= & B_{S_{5}}\left(2 J_{2} S_{8}+2 J_{2} S_{6}+4 J_{3} S_{7}\right. \\
& \left.+2 J_{4}\left(S_{1}+S_{2}+S_{3}+S_{4}\right)\right),
\end{aligned}
$$

где $B_{S_{i}}$ - функция Бриллюэна. Выражения для $\left\langle S_{2}\right\rangle$, $\left\langle S_{3}\right\rangle,\left\langle S_{4}\right\rangle$ получаются из выражения для $\left\langle S_{2}\right\rangle$ путем замены индексов $(1 \rightarrow 2,4 \rightarrow 3,2 \rightarrow 1,3 \rightarrow 4),(1 \rightarrow 3$,
$4 \rightarrow 2,2 \rightarrow 4,3 \rightarrow 1),(1 \rightarrow 4,4 \rightarrow 1,2 \rightarrow 3,3 \rightarrow 2)$ coответственно. Для $\left\langle S_{6}\right\rangle,\left\langle S_{7}\right\rangle,\left\langle S_{8}\right\rangle$ должна производиться замена индексов в выражении для $\left\langle S_{5}\right\rangle:(5 \rightarrow 6,8 \rightarrow 7$, $6 \rightarrow 5,7 \rightarrow 8),(5 \rightarrow 7,8 \rightarrow 6,6 \rightarrow 8,7 \rightarrow 5),(5 \rightarrow 8$, $8 \rightarrow 5,6 \rightarrow 7,7 \rightarrow 6$ ) соответственно.

Для оценки температуры фазового перехода функции Бриллюэна в описанной выше системе уравнений для средних намагниченностей подрешеток раскладывались в ряд, и составлялось детерминантное уравнение. Наибольшее по величине решение этого уравнения, которое и было выбрано за температуру фазового перехода в ферромагнитное состояние, составило $T_{c} \approx 1 \mathrm{~K}$. Столь низкая температура перехода обусловлена как малыми значениями обменных констант, полученных в данном расчете, так и наличием конкуренции между ними.

В работе [3] проводились измерения обратной магнитной восприимчивости тетрабората европия в интервале температур от 2 до $24 \mathrm{~K}$. Магнитного упорядочения в этом диапазоне температур авторами обнаружено не было, в результате чего был сделан вывод о том, что данное соединение является парамагнетиком, при этом температурная зависимость обратной восприимчивости в указанной работе не приводится. В [3] приводится экспериментальное измеренное значение магнитного момента для иона европия $\mu_{\mathrm{Eu}}^{\exp }=8.02 \mu_{\mathrm{B}}$, что хорошо согласуется с вычисленным здесь $\left(\mu_{\mathrm{Eu}}^{\mathrm{calc}}=6.94 \mu_{\mathrm{B}}\right)$.

Как отмечалось выше, кристалл тетрабората европия является пироэлектриком и обладает структурой с полярной группой симметрии $P m n 2_{1}$ с направлением электрической поляризации вдоль оси $z$. Поскольку EBO содержит магнитный ион $\mathrm{Eu}^{2+}$, можно ожидать в данном соединении магнитоэлектрический эффект. Исследование магнитоэлектрических свойств в магнитных пироэлектриках представляет интерес, так как в этих соединениях можно ожидать достаточно больших величин магнитоэлектрического эффекта (см., например, [20]). Для оценки величины изменения поляризации в кристалле тетрабората европия при возникновении магнитного порядка использовались набор равновесных координат, полученный при релаксации структуры без учета спиновой поляризации, и набор равновесных координат, полученный при релаксации структуры с учетом ферромагнитного упорядочения (параметры элементарной

Таблица 4. Изменение относительных координат ионов при ферромагнитном упорядочении, номинальные заряды ионов (выражение в зарядах электрона $e$ ) и изменение поляризации

\begin{tabular}{c|c|c|c|c|c|c|c|c}
\hline \multirow{2}{*}{ Атом } & \multirow{2}{*}{$\begin{array}{c}\text { Позиция } \\
\text { Уайкова }\end{array}$} & \multirow{2}{*}{$\begin{array}{c}\text { Заряд, } \\
\end{array}$} & \multicolumn{2}{|c|}{ Изменение относительных координат атомов } & \multicolumn{3}{|c}{ Изменение поляризации, $\mu \mathrm{C} / \mathrm{m}^{2}$} \\
\cline { 4 - 8 } & & $\Delta x / a$ & $\Delta y / b$ & $\Delta z / c$ & $\Delta P_{x}$ & $\Delta P_{y}$ & $\Delta P_{z}$ \\
\hline $\mathrm{Eu}$ & $2 a$ & +2 & 0.00000 & 0.00993 & 0.00380 & & & \\
$\mathrm{~B} 1$ & $4 b$ & +3 & -0.00024 & 0.00064 & -0.00079 & & \\
$\mathrm{~B} 2$ & $4 b$ & +3 & -0.00016 & 0.00016 & -0.00013 & & \\
$\mathrm{O} 1$ & $2 a$ & -2 & 0.00000 & -0.00100 & 0.00245 & 0 & 0 & 3973 \\
$\mathrm{O} 2$ & $4 b$ & -2 & 0.00006 & -0.00049 & -0.00051 & & & \\
$\mathrm{O} 3$ & $4 b$ & -2 & -0.00040 & 0.00065 & -0.00113 & & & \\
$\mathrm{O} 4$ & $4 b$ & -2 & 0.00050 & 0.00079 & -0.00057 & & &
\end{tabular}


ячейки в обоих расчетах соответствовали равновесным параметрам ячейки расчета с учетом спиновой поляризации). Полученные величины смещений ионов приведены в табл. 4. Изменение поляризации, возникающее в результате магнитоэлектрического эффекта, вычислялось по формуле

$$
\Delta P_{i}=\frac{1}{V} \sum_{j=1}^{N} Z_{j}^{i} u_{j}^{i},
$$

где индекс $i=x, y, z ; V$ - объем ячейки; $N$ - число атомов в ячейке; $Z_{j}^{i}-$ номинальный заряд иона с номером $j ; u_{j}^{i}$ - смещение иона с номеро $i$. При ферромагнитном упорядочении параллельно оси $z$ кристалла симметрия структуры ЕВО не изменяется, полярной осью является ось $z$. Действительно, ненулевым оказалось изменение поляризации только вдоль оси $z$, именно в этом направлении происходят полярные смещения ионов. Полученное значение $\Delta P_{z}=3973 \mu \mathrm{C} / \mathrm{m}^{2}$ является довольно большой величиной магнитоэлектрического эффекта. Для сравнения можно указать на результаты работы в которой [20], проведены экспериментальное и теоретическое исследования магнитоэлектрического эффекта в пироэлектрике $\mathrm{Ni}_{3} \mathrm{TeO}_{6}$. Установленную в [20] величину индуцированной магнитным полем поляризации $\Delta P \approx 3000 \mu \mathrm{C} / \mathrm{m}^{2}$ авторы называют самой большой среди полученных для магнитных пироэлектриков.

\section{8. Заключение}

Перечислим основные результаты работы.

Были вычислены частоты колебаний кристаллической решетки EBO в центре зоны Бриллюэна. Полученные значения частот хорошо согласуются с имеющимися экспериментальными данными. Был проведен расчет электронной зонной структуры, построены дисперсионные кривые и плотность электронных состояний. Полученная ширина запрещенной зоны составила приблизительно $4 \mathrm{eV}$. Рассчитана зависимость коэффициента поглощения от длины волны, из которой видно наличие двух максимумов при длинах волн 240 и $280 \mathrm{~nm}$. Эти данные согласуются с результатами эксперимента по исследованию поглощения изоструктурного кристалла $\mathrm{SrB}_{4} \mathrm{O}_{7}$, допированного $5 \% \mathrm{Eu}^{+2}$, в котором наблюдаются пики поглощения света при длинах волн приблизительно 240 и $300 \mathrm{~nm}$. При этом по экспериментальным данным для пропускания света в недопированном кристалле $\mathrm{SrB}_{4} \mathrm{O}_{7}$ в диапазоне длин волн от 130 до $300 \mathrm{~nm}$ таких пиков поглощения обнаружено не было, и можно сделать вывод о том, что в случае допированного $\mathrm{SrB}_{4} \mathrm{O}_{7}$ появление пиков поглощения обусловлено наличием ионов европия. Расчет магнитных свойств кристалла ЕВО показал, что основным состоянием является состояние с ферромагнитным упорядочением спинов $\mathrm{Eu}^{2+}$. Вычисленные значения обменных констант составили $J_{1}=-1.4 \cdot 10^{-5} \mathrm{eV}$, $J_{2}=3.9 \cdot 10^{-5} \mathrm{eV}, J_{3}=5.3 \cdot 10^{-5} \mathrm{eV}, J_{4}=0.7 \cdot 10^{-5} \mathrm{eV}$.
В рамках приближения молекулярного поля была проведена оценка температуры фазового перехода в ферромагнитное состояние, которая составила $T_{c} \approx 1 \mathrm{~K}$. Столь низкая температура перехода обусловлена как малыми значениями полученных в данном расчете обменных констант, так и наличием конкуренции между ними. Исследуемый кристалл тетрабората европия является магнитным пироэлектриком и, следовательно, может проявлять магнитоэлектрические свойства. Оценка изменения электрической поляризации при ферромагнитном упорядочении дала величину $\Delta P_{z}=3973 \mu \mathrm{C} / \mathrm{m}^{2}$.

\section{Список литературы}

[1] Yu.S. Oseledchik, A.L. Prosvirnin, A.I. Pisarevskiy, V.V. Starshenko, V.V. Osadchuk, S.P. Belokrys, N.V. Svitanko, A.S. Korol, S.A. Krikunov, A.F. Selevich. Opt. Mater. 4, 669 (1995).

[2] A.I. Zaitsev, A.S. Aleksandrovskii, A.V. Zamkov, A.M. Sysoev. Inorg. Mater. 42, 1360 (2006).

[3] K. Machida, H. Hata, K. Okuno, G. Adachi. J. Shiokawa. J. Inorg. Nucl. Chem. 41, 1425 (1979).

[4] K. Machida, G. Adachi, J. Shiokawa. Acta Cryst. B 36, 2008 (1980).

[5] G. Kresse, J. Hafner. Phys. Rev. B 47, 558 (1993).

[6] G. Kresse, J. Furthmuller. Phys. Rev. B 54, 11169 (1996).

[7] G. Kresse, D. Joubert. Phys. Rev. B 59, 1758 (1999).

[8] P.E. Blöchl. Phys. Rev. B 50, 17953 (1994).

[9] J.P. Perdew. In: Electronic Structures of Solids'91 / Eds P. Ziesche, H. Eschrig. Akademie Verlag, Berlin (1991). P. 11.

[10] H.J. Monkhorst, J.D. Pack. Phys. Rev. B 13, 5188 (1976).

[11] S.L. Dudarev, G.A. Botton, S.Y. Savrasov, C.J. Humphreys, A.P. Sutton. Phys. Rev. B 57, 1505 (1998).

[12] X. Hu, J. Wang, B. Teng, C.-K. Loong, M. Grimsditch. J. Appl. Phys. 97, 033501 (2005).

[13] В.И. Зиненко, М.С. Павловский, А.И. Зайцев, А.С. Крылов, А.С. Шинкоренко. ЖЭТФ 142, 511 (2012).

[14] Y. Wang, M. Feng, H. Wang, P. Fu, J. Wang, X. Cao, G. Lan. J. Phys.: Condens. Matter 19, 436207 (2007).

[15] J.M. An, K.D. Belashchenko. Phys. Rev. B 88, 054421 (2013).

[16] D.B. Ghosh, M. De, S.K. De. Phys. Rev. B 70, 115211 (2004).

[17] M. Gajdos̆, K. Hummer, G. Kresse, J. Furthmüller, F. Bechstedt. Phys. Rev. B 73, 045112 (2006).

[18] Z. Jiao, S. Li, Q. Yan, X. Wang, D. Shen. J. Phys. Chem. Solids 72, 252 (2011).

[19] V. Petrov, F. Noack, D. Shen, F. Pan, G. Shen, X. Wang, R. Komatsu, V. Alex. Opt. Lett. 29, 4, 373 (2004).

[20] J.W. Kim, S. Artyukhin, E.D. Mun, M. Jaime, N. Harrison, A. Hansen, J.J. Yang, Y.S. Oh, D. Vanderbilt, V.S. Zapf, S.-W. Cheong. Phys. Rev. Lett. 115, 137201 (2015). 